\title{
Bounds for the Density of Abundant Integers
}

\author{
Marc Deléglise
}

\section{CONTENTS}

Introduction

1. Expressing $A(x)$ as a Sum

2. Trivial Bounds for $A_{k}(x)$

3. Lower Bound for $A(x)$

4. Upper Bounds for $A_{k}(x)$

5. Mean Values of $f(n)^{r}$ and Upper Bounds for $A_{k}(x)$

6. Upper Bounds for the Euler Products $\Lambda_{k}(r)$

7. Numerical Results

8. Other Experimental Results

References
We say that an integer $\mathrm{n}$ is abundant if the sum of the divisors of $n$ is at least 2n. It has been known [Wall 1972] that the set of abundant numbers has a natural density $\mathrm{A}(2)$ and that $0.244<\mathrm{A}(2)<0.291$. We give the sharper bounds

$$
0.2474<\mathrm{A}(2)<0.2480 \text {. }
$$

\section{INTRODUCTION}

Let $x$ be a positive real number, and $n$ an integer. Let $\sigma(n)$ be the sum of the divisors of $n$, and set

$$
f(n)=\frac{\sigma(n)}{n}, \quad \mathscr{A}(x)=\{n: f(n) \geq x\} .
$$

A number in $\mathscr{A}(x)$ is called $x$-abundant, or simply abundant if $x=2$.

Davenport proved that $\mathscr{A}(x)$ has a natural density $A(x)$, and that $A(x)$ is a continuous function of $x$; see, for example, [Davenport 1933; Elliott 1979, Chapter 5; Tenenbaum 1995, III.1 and III.2].

Behrend [1933] proved that $0.241<A(2)<0.314$, and Wall [1972] improved this to $0.244<A(2)<$ 0.291 . We prove here the following:

Theorem 0.1. The density $A(2)$ of the set of abundant numbers satisfies

$$
0.2474<A(2)<0.2480 \text {. }
$$

This answers a question asked by Henri Cohen: Is the proportion of abundant numbers more or less than a quarter? The method used is essentially that given by Behrend, the computer allowing us to do more computations. This method in fact gives the density $A(x)$ for every $x$.

Perhaps it could be worthwile to try an analytic method. Cohen, Deshouillers, Martinet showed in 
[Martinet et al. 1973] that the Mellin transform of $A(x)$ is the function

$$
g(s)=\frac{1}{s} \prod_{p \geq 2} \frac{1}{\left(1-\frac{1}{p}\right)^{s-1}} \sum_{l \geq 0} \frac{1}{p^{l}}\left(1-\frac{1}{p^{l+1}}\right)^{s} .
$$

Hence, by inversion, we have for every $\sigma>1$

$$
A(x)=\frac{1}{2 i \pi} \int_{\sigma-i \infty}^{\sigma+i \infty} x^{-s} g(s) d s,
$$

but the computation of this integral seems to be difficult; taking $x=2$ and $\sigma=2$ we computed the sum between $2-10000 i$ and $2+10000 i$, and got the approximate value 0.242 . For large values of $\operatorname{Im}(s)$ the computation of $g(s)$ is difficult.

\section{EXPRESSING $A(x)$ AS A SUM}

We denote by $\left(p_{n}\right)_{n \geq 1}$ the increasing sequence of primes. Let $k$ be a fixed integer. We consider the set

$$
\mathscr{A}_{k}(x)=\left\{n: f(n) \geq x, \operatorname{gcd}\left(n, p_{1} p_{2} \ldots p_{k}\right)=1\right\} .
$$

This set has a density [Elliott 1979; Tenenbaum 1995], which will be denoted by $A_{k}(x)$.

Let $n$ be an arbitrary integer. We denote by $n_{1}$ the product of the prime factors of $n$ among $\left\{p_{1}, p_{2}, \ldots, p_{k}\right\}$ and we write $n=n_{1} n_{2}$. The function $f$ is multiplicative and $f(n)=f\left(n_{1}\right) f\left(n_{2}\right)$ is greater than or equal to $x$ if and only if $f\left(n_{2}\right) \geq$ $x / f\left(n_{1}\right)$. This proves that $\mathscr{A}(x)$ is partitioned as follows:

$$
\mathscr{A}(x)=\bigcup_{n_{1}=p_{1}^{\alpha_{1} \ldots p_{k}}} n_{1} \mathscr{A}_{k}\left(\frac{x}{f\left(n_{1}\right)}\right) .
$$

Considering the densities we have:

\section{Proposition 1.1.}

$$
A(x)=\sum_{n_{1}=p_{1}^{\alpha_{1}} \ldots p_{k}^{\alpha_{k}}} \frac{1}{n_{1}} A_{k}\left(\frac{x}{f\left(n_{1}\right)}\right),
$$

where the sum is taken over all $n_{1}$ that are a product of primes belonging to $\left\{p_{1}, p_{2}, \ldots, p_{k}\right\}$.
To see this, it is sufficient to prove the following lemma.

Lemma 1.2. Let $p$ be an integer greater than 1 and $\left(A_{\alpha}\right)_{\alpha \geq 0}$ a sequence of disjoint sets having densities $d_{\alpha}$. Set $\mathscr{A}=\bigcup_{\alpha \geq 0} p^{\alpha} \mathscr{A}_{\alpha}$. Then $\mathscr{A}$ has a density $d(\mathscr{A})$ and

$$
d(\mathscr{A})=\sum_{\alpha \geq 0} \frac{1}{p^{\alpha}} d_{\alpha}
$$

Proof. Write

$$
\mathscr{A}=\left(\bigcup_{0 \leq \alpha \leq r} p^{\alpha} \mathscr{A}_{\alpha}\right) \cup\left(\bigcup_{\alpha>r} p^{\alpha} \mathscr{A}_{\alpha}\right)
$$

The second set in this union is formed of multiples of $p^{r+1}$. Its upper density is bounded by $1 / p^{r+1}$ and

$$
\sum_{0 \leq \alpha \leq r} \frac{1}{p^{\alpha}} d_{\alpha} \leq \underline{d}(\mathscr{A}) \leq \bar{d}(\mathscr{A}) \leq \sum_{0 \leq \alpha \leq r} \frac{1}{p^{\alpha}} d_{\alpha}+\frac{1}{p^{r+1}}
$$

where $\underline{d}$ and $\bar{d}$ denote the lower and upper densities. We let $r \rightarrow \infty$ and we get the result.

\section{TRIVIAL BOUNDS FOR $A_{k}(x)$}

Proposition 2.1. For every $k \geq 0$ and every $x>0$ we have

$$
A_{k}(x) \leq F_{k}
$$

and

$$
A_{k}(x)=F_{k} \quad \text { if } \quad x \leq 1,
$$

where $F_{k}=\prod_{i=1}^{k}\left(1-1 / p_{i}\right)$.

Proof. Clear, since $\mathscr{A}_{k}(x)$ is formed only with integers coprime with $p_{1} p_{2} \ldots p_{k}$, and comprises all these integers if $x \leq 1$.

\section{LOWER BOUND FOR $\mathrm{A}(\mathrm{x})$}

Let $z$ be a arbitrary positive real parameter. If in (1-2) we just keep the integers $n_{1}=p_{1}^{\alpha_{1}} \ldots p_{k}^{\alpha_{k}} \leq z$, we get a lower bound for $A(x)$. Hence

$$
A(x) \geq \sum_{n_{1}=p_{1}^{\alpha_{1}} \ldots p_{k}^{\alpha_{k}}}^{n_{1} \leq z} \frac{1}{n_{1}} A_{k}\left(\frac{x}{f\left(n_{1}\right)}\right) .
$$


We still get a lower bound if we just keep those $n_{1}$ such that $f\left(n_{1}\right) \geq x$; hence

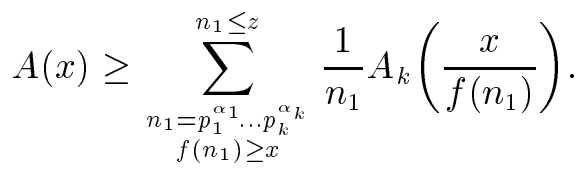

By $(2-2)$, all the $A_{k}\left(x / f\left(n_{1}\right)\right)$ are equal to $F_{k}$; hence

$$
A(x) \geq F_{k} \sum_{\substack{n_{1}=p_{1} \ldots p_{k} \\ f\left(n_{1}\right) \geq x}}^{n_{1} \leq z} \frac{1}{n_{1}} .
$$

This lower bound is almost trivial and could have been shown slightly differently. We choose an upper bound $z$ and a set $\left\{p_{1}, p_{2}, \ldots, p_{k}\right\}$ of small primes. We compute all the integers $m$ less than $z$, composed of prime factors from $\left\{p_{1}, p_{2}, \ldots, p_{k}\right\}$, and $x$-abundant. Every multiple of an abundant number being abundant, all the products of the numbers $m$ thus obtained by some prime factors out of $\left\{p_{1}, p_{2}, \ldots, p_{k}\right\}$ are still abundant numbers. The lower bound for $A(x)$ is the density of this set, $F_{k} \sum_{m} 1 / m$.

\section{UPPER BOUNDS FOR $A_{k}(x)$}

As in the previous section, we introduce a real positive parameter $z$ and write

$$
\begin{aligned}
A(x)=\sum_{n_{1}=p_{1}^{\alpha_{1} \ldots p_{k}^{\alpha}}}^{n_{1} \leq z} \frac{1}{n_{1}} A_{k}\left(\frac{x}{f\left(n_{1}\right)}\right) & \\
& +\sum_{n_{1}=p_{1}^{\alpha_{1} \ldots p_{k}^{\alpha}}}^{z<n_{1}} \frac{1}{n_{1}} A_{k}\left(\frac{x}{f\left(n_{1}\right)}\right) .
\end{aligned}
$$

In the second sum, each value of $A_{k}$ is bounded from above by $F_{k}$; thus the second sum is bounded from above by

$$
\begin{aligned}
F_{k} \sum_{n_{1}=p_{1}^{\alpha_{1} \ldots p_{k}^{\alpha_{k}}}}^{z<n_{1}} \frac{1}{n_{1}} & =F_{k} \sum_{n_{1}=p_{1}^{\alpha_{1} \ldots p_{k}^{\alpha_{k}}}}^{1 \leq n_{1} \leq \infty} \frac{1}{n_{1}}-F_{k} \sum_{n_{1}=p_{1}^{\alpha_{1} \ldots p_{k}^{\alpha_{k}}}}^{n_{1} \leq z} \frac{1}{n_{1}} \\
& =1-F_{k} \sum_{n_{1}=p_{1}^{\alpha_{1} \ldots p_{k}^{\alpha_{k}}}}^{n_{1} \leq z} \frac{1}{n_{1}}
\end{aligned}
$$

so

$$
\begin{aligned}
A(x) \leq \sum_{n_{1}=p_{1}^{\alpha_{1} \ldots p_{k} \alpha_{k}}}^{n_{1} \leq z} \frac{1}{n_{1}} A_{k}\left(\frac{x}{f\left(n_{1}\right)}\right) & \\
& +1-F_{k} \sum_{n_{1}=p_{1}^{\alpha_{1} \ldots p_{k}}{ }_{k}^{n_{1} \leq z}}^{n_{k}} \frac{1}{n_{1}} .
\end{aligned}
$$

It remains to bound the values of $A_{k}$ that appear in the sum (4-1). If we just use the trivial upper bound $A_{k} \leq F_{k}$ we will get $A(x) \leq 1$, so we need a nontrivial upper bound for $A_{k}(x)$. This is the subject of the next section.

\section{MEAN VALUES OF $\mathrm{f}(\mathrm{n})^{\mathrm{r}}$ AND UPPER BOUNDS FOR $A_{k}(x)$}

Let $f_{k}$ be the multiplicative function that takes the value 1 for $p^{\alpha}$ with $p \leq p_{k}$ and the value $f\left(p^{\alpha}\right)$ for $p>p_{k}$. We fix an integer $r$ and we consider $g=f_{k}^{r}$ and the mean value of $g$ computed on the first $n$ integers:

$$
M_{n}=\frac{1}{n} \sum_{m=1}^{n} g(m) .
$$

Let $\rho$ be the convolution product of $g$ and the Möbius $\mu$ function:

$$
\rho(m)=\sum_{d \mid m} \mu\left(\frac{m}{d}\right) g(d) .
$$

The Möbius inversion formula gives

$$
\begin{aligned}
M_{n} & =\frac{1}{n} \sum_{m=1}^{n} g(n)=\frac{1}{n} \sum_{m=1}^{n} \sum_{d \mid m} \rho(d) \\
& =\frac{1}{n} \sum_{d=1}^{n} \rho(d)\left[\frac{n}{d}\right] \leq \frac{1}{n} \sum_{d=1}^{n} \rho(d) \frac{n}{d} \\
& \leq \sum_{d=1}^{\infty} \frac{\rho(d)}{d}=\Lambda_{k}(r) .
\end{aligned}
$$

The function $\rho(d) / d$ is multiplicative, so $\Lambda_{k}(r)$ is also equal to the value of the Euler product

$$
\Lambda_{k}(r)=\prod_{p}\left(1+\frac{\rho(p)}{p}+\frac{\rho\left(p^{2}\right)}{p^{2}}+\cdots+\right) .
$$


Using the definition equation (5-1) of $\rho$, we have

$$
\begin{aligned}
\rho\left(p^{\alpha}\right) & =g\left(p^{\alpha}\right)-g\left(p^{\alpha-1}\right) \\
& =\left(1+\frac{1}{p}+\cdots+\frac{1}{p^{\alpha}}\right)^{r}-\left(1+\frac{1}{p}+\cdots+\frac{1}{p^{\alpha-1}}\right)^{r}
\end{aligned}
$$

when $p>p_{k}$ and $\alpha>0$, otherwise $\rho\left(p^{\alpha}\right)=0$.

We return to the sum

$$
n M_{n}=\sum_{m=1}^{n} g(m) .
$$

Let $B_{n}$ be the number of integers $m$ between 1 and $n$ such that $f_{k}(m) \geq x$, or equivalently $g(m) \geq x^{r}$. We collect the terms of this sum in two classes, first those terms for which $g(m) \geq x^{r}$, that are bounded from below by $x^{r}$, and the other terms, that are bounded from below by 1 . We get

$$
x^{r} B_{n}+n-B_{n} \leq n M_{n} \leq n \Lambda_{k}(r) ;
$$

dividing by $n$ and letting $n \rightarrow \infty$ we get

$$
B_{k}(x) \leq \frac{\Lambda_{k}(r)-1}{x^{r}-1},
$$

where $B_{k}(x)$ is the density of the set of all $m$ such that $f_{k}(m) \geq x$. This set is the disjoint union of the $p_{1}^{\alpha_{1}} \ldots p_{k}^{\alpha_{k}} \mathscr{A}_{k}(x)$, and we deduce the following upper bound, proved by Behrend [1933].

Proposition 5.1. For every integer $r \geq 1$ and every $k$,

$$
A_{k}(x) \leq F_{k} \frac{\Lambda_{k}(r)-1}{x^{r}-1} .
$$

Table 1 gives the upper bounds for $\Lambda_{95}(r)-1$ for $r=1,2,4,8,16, \ldots, 4096$.

\begin{tabular}{|rl|rl|}
\hline$r$ & $\Lambda_{95}(r)-1 \leq$ & $r$ & $\Lambda_{95}(r)-1 \leq$ \\
\hline 1 & 0.000284 & 64 & 0.0189 \\
2 & 0.000568 & 128 & 0.0395 \\
4 & 0.00114 & 256 & 0.0866 \\
8 & 0.00228 & 512 & 0.213 \\
16 & 0.00458 & 1024 & 0.726 \\
32 & 0.00925 & 2048 & 12.3 \\
& & 4096 & $1.37 \times 10^{17}$ \\
\hline
\end{tabular}

TABLE 1. Upper bounds for $\Lambda_{95}(r)-1$.
When $x$ is very close to 1 , almost every integer is $x$-abundant and the trivial upper bound $(2-1)$ is better than the upper bound (5-3). Table 2 shows this phenomenon. It gives for some values of $x$ the best upper bound for $A_{k}(x)$ obtained by formula (5-3) choosing the right value for $r$. The value $r=$ 0 on the first line means that, for this $x=1.0001$, the trivial upper bound (2-1) is the better one.

\begin{tabular}{|rrc|rrl|}
\hline$x$ & $r$ & $A_{95}(x) \leq$ & $x$ & $r$ & $A_{95}(x) \leq$ \\
\hline 1.0001 & 0 & 0.0897 & 1.005 & 2048 & $4.35 \times 10^{-5}$ \\
1.001 & 1 & 0.0254 & 1.01 & 2048 & $1.68 \times 10^{-9}$ \\
1.002 & 1024 & 0.0096 & 1.02 & 4096 & $9.21 \times 10^{-20}$ \\
\hline
\end{tabular}

TABLE 2. Some upper bounds for $A_{95}(r)$ obtained using Table 1.

\section{UPPER BOUNDS FOR THE EULER PRODUCTS $\Lambda_{\mathrm{k}}(\mathrm{r})$}

In this section we give some effective upper bounds used to get upper bounds for the Euler products $\Lambda_{k}(r)$. In all this section we write

$$
\begin{aligned}
\rho\left(p^{\alpha}\right) & =\left(1+\frac{1}{p}+\cdots+\frac{1}{p^{\alpha}}\right)^{r}-\left(1+\frac{1}{p}+\cdots+\frac{1}{p^{\alpha-1}}\right)^{r} \\
& =\sum_{d \mid p^{\alpha}} \mu(d)\left(f\left(\frac{p^{\alpha}}{d}\right)\right)^{r} .
\end{aligned}
$$

This is the $\rho$ function defined by $(5-1)$ for $k=0$.

We gave in [Deléglise and Nicolas 1994] a method to quickly compute a good approximate value of an Euler product $\prod_{p} g(1 / p)$, when $g$ is a holomorphic function around 0 whose first Taylor series coefficients are not too large. This method could have been used to get some very accurate values for the first $\Lambda_{k}(r)$. For very large values of $r$ the accuracy would not be so good. Since we just need an upper bound for each $\Lambda_{k}(r)$, we will just use the trivial method: find upper bounds for the partial products, and for the tails of the products.

Lemma 6.1. Let $r$ be an integer $\geq 1$ and $p \geq 2 r$. Then

$$
\left(1+\frac{1}{p}\right)^{r}-1<1.3 \frac{r}{p}
$$


Proof. We have

$$
\begin{aligned}
\frac{(1+1 / p)^{r}-1}{r / p} & =\frac{\exp (r \ln (1+1 / p))-1}{r / p} \\
& <\frac{\exp (r / p)-1}{r / p} \\
& \leq \frac{e^{1 / 2}-1}{1 / 2}<1.3 .
\end{aligned}
$$

Lemma 6.2. Let $r$ an integer $\geq 2$ and $p \geq 2 r$. Then

$$
\left(\frac{1}{1-1 / p}\right)^{r-1}<\frac{16}{9}<1.78
$$

Proof. Let $u=1 / p$. Then

$$
y=\left(\frac{1}{1-1 / p}\right)^{r-1}<\left(\frac{1}{1-1 / p}\right)^{r} \leq\left(\frac{1}{1-u}\right)^{1 / 2 u}
$$

hence

$$
\ln (y)=\frac{1}{2 u} \ln \left(\frac{1}{1-u}\right) \leq 2 \ln \left(\frac{1}{1-\frac{1}{4}}\right)=\ln \frac{16}{9},
$$

since the function $(1 / u) \ln (1 /(1-u))$ is increasing for $0<u \leq \frac{1}{2 r} \leq \frac{1}{4}$.

Lemma 6.3. For every integer $r$ and every prime $p$,

$$
\begin{aligned}
& \sum_{\alpha \geq 0} \frac{\rho\left(p^{\alpha}\right)}{p^{\alpha}} \\
& \quad \leq 1+\frac{(1+1 / p)^{r}-1}{p}+r\left(\frac{1}{1-1 / p}\right)^{r-1} \frac{1}{p^{4}-p^{2}} .
\end{aligned}
$$

Proof. Set

$$
Y=1+\frac{1}{p}+\cdots+\frac{1}{p^{\alpha-1}}, \quad X=Y+\frac{1}{p^{\alpha}} .
$$

We get, for $\alpha \geq 1$,

$$
\begin{aligned}
\frac{\rho\left(p^{\alpha}\right)}{p^{\alpha}} & =\frac{1}{p^{\alpha}}\left(X^{r}-Y^{r}\right) \\
& =\frac{1}{p^{2 \alpha}}\left(X^{r-1}+X^{r-2} Y+\cdots+Y^{r-1}\right) \\
& \leq \frac{r}{p^{2 \alpha}} X^{r-1} \leq \frac{r}{p^{2 \alpha}}\left(\frac{1}{1-1 / p}\right)^{r-1}
\end{aligned}
$$

Using this upper bound for $\alpha \geq 2$ in the sum

$$
\sum_{\alpha \geq 0} \frac{\rho\left(p^{\alpha}\right)}{p^{\alpha}}
$$

we get the conclusion.

Lemma 6.4. For every integer $r \geq 1$ and every $p \geq$ $\max (2 r, 15)$ we have

$$
\sum_{\alpha \geq 0} \frac{\rho\left(p^{\alpha}\right)}{p^{\alpha}}<1+1.31 \frac{r}{p^{2}} .
$$

Proof. The preceding three lemmas give, for every $r \geq 2$,

$$
\begin{aligned}
\sum_{\alpha \geq 0} \frac{\rho\left(p^{\alpha}\right)}{p^{\alpha}} & \leq 1+1.3 \frac{r}{p^{2}}+1.78 \frac{r}{p^{4}-p^{2}} \\
& =1+\frac{r}{p^{2}}\left(1.3+1.78 \frac{1}{p^{2}-1}\right) \\
& \leq 1+1.31 \frac{r}{p^{2}} \quad \text { if } p \geq 15 .
\end{aligned}
$$

For $r=1$ this upper bound is still true, because

$$
\sum_{\alpha \geq 0} \frac{\rho\left(p^{\alpha}\right)}{p^{\alpha}}=\sum_{\alpha \geq 0} \frac{1}{p^{2 \alpha}}=1+\frac{1}{p^{2}-p^{4}} .
$$

Lemma 6.5. For every integer $r$ with $1 \leq r \leq 10000$ we have

$$
\prod_{p>10^{6}}\left(\sum_{\alpha \geq 0} \frac{\rho\left(p^{\alpha}\right)}{p^{\alpha}}\right) \leq 1+\frac{r}{10^{7}}
$$

Proof. Set

$$
u=\prod_{p>10^{6}}\left(\sum_{\alpha \geq 0} \frac{\rho\left(p^{\alpha}\right)}{p^{\alpha}}\right) .
$$

Using Lemma 6.4 we get

$$
\ln (u) \leq 1.31 r \sum_{p>10^{6}} \frac{1}{p^{2}} .
$$

The sum of $1 / p^{2}$ can be computed as explained in [Deléglise and Nicolas 1994, pp. 331-332], or it can be found in [Glaisher 1891]:

$$
\sum_{p} \frac{1}{p^{2}}=0.452247420041 \ldots \text {. }
$$




\begin{tabular}{|c|c|c|c|c|c|c|c|}
\hline \multicolumn{2}{|l|}{ Interval } & \multicolumn{2}{|l|}{ Interval } & \multicolumn{2}{|l|}{ Interval } & \multicolumn{2}{|l|}{ Interval } \\
\hline$\left[1,10^{1}\right]$ & 1 & {$\left[1,10^{6}\right]$} & 24799 & {$\left[10^{9}, 10^{9}+10^{7}\right)$} & 2476049 & {$\left[10^{14}, 10^{14}+10^{7}\right)$} & 2476150 \\
\hline$\left[1,10^{2}\right]$ & 24 & {$\left[1,10^{7}\right]$} & 2476741 & {$\left[10^{10}, 10^{10}+10^{7}\right)$} & 2476372 & {$\left[10^{15}, 10^{15}+10^{7}\right)$} & 2476212 \\
\hline$\left[1,10^{3}\right]$ & 249 & {$\left[1,10^{8}\right]$} & 24760673 & {$\left[10^{11}, 10^{11}+10^{7}\right)$} & 2476154 & {$\left[10^{16}, 10^{16}+10^{7}\right)$} & 2476247 \\
\hline$\left[1,10^{4}\right]$ & 2492 & {$\left[1,10^{9}\right]$} & 247610965 & {$\left[10^{12}, 10^{12}+10^{7}\right)$} & 2476199 & {$\left[10^{17}, 10^{17}+10^{7}\right)$} & 2476098 \\
\hline & & & & {$\left[10^{13}, 10^{13}+10^{7}\right)$} & 2476213 & {$\left[10^{18}, 10^{18}+10^{7}\right)$} & 2476304 \\
\hline
\end{tabular}

TABLE 3. Frequency of abundant numbers in different intervals.

Hence, subtracting $\sum_{p \leq 10^{6}} 1 / p^{2}$, we have

$$
\sum_{p>10^{6}} \frac{1}{p^{2}}=0.0000000677 \ldots
$$

and

$$
\ln (u)<0.9 \frac{r}{10^{7}}<10^{-3}
$$

and finally

$$
u=e^{\ln u}<1+\frac{r}{10^{7}},
$$

using the estimate $e^{t}<1+\frac{10}{9} t$ for $t<0.001$.

We get an upper bound for the Euler product (5-2), writing

$$
\begin{aligned}
& \prod_{p>p_{k}}\left(\sum_{\alpha \geq 0} \frac{\rho\left(p^{\alpha}\right)}{p^{\alpha}}\right) \\
& \quad=\prod_{p_{k}<p \leq 10^{6}}\left(\sum_{\alpha \geq 0} \frac{\rho\left(p^{\alpha}\right)}{p^{\alpha}}\right) \prod_{p>10^{6}}\left(\sum_{\alpha \geq 0} \frac{\rho\left(p^{\alpha}\right)}{p^{\alpha}}\right) .
\end{aligned}
$$

The first product is bounded by Lemma 6.3 and the second by Lemma 6.5 .

Table 1 gives the upper bounds for $\Lambda_{95}(r)-1$ for $r=1,2,4,8,16, \ldots, 4096$. These are the values used for bounding the values $A_{k}$ that appear in formula (4-1).

\section{NUMERICAL RESULTS}

We have bounded $A(2)$ using (3-1) and (4-1) with $x=2, k=95$ (which is the number of primes less than 500), and $z=10^{14}$. For the upper estimate each term

$$
A_{k}\left(\frac{x}{f\left(p_{1}^{\alpha_{1}} \ldots p_{k}^{\alpha_{k}}\right)}\right)
$$

in (4-1) is bounded using formula (5-3) with $r=$ $1,2,4,8, \ldots, 4096$ and the trivial bound $(2-1)$; we keep the best result obtained. This requires the enumeration of all the $p_{1}^{\alpha_{1}} \ldots p_{k}^{\alpha_{k}}$ not greater than $z$, which is done by a backtracking procedure. The total number of these $n$ less than $10^{14}$ whose prime factors are less than 500 is 23581230171 .

The computation was performed on an HP900730 workstation, using about 100 hours of CPU time. It yields

$$
0.2474<A(2)<0.2480 ;
$$

in particular $A(2)=0.247 \ldots$.

\section{OTHER EXPERIMENTAL RESULTS}

We computed the number of abundant numbers less than $N$ for $N=1,10,10^{2}, \ldots, 10^{9}$, and also the number of abundant numbers in the intervals $\left[N, N+10^{7}\right)$ for $N=10^{9}, 10^{10}, \ldots, 10^{18}$. The results are given in Table 3 and seem to show that the next digit of $A(2)$ is a 6 .

We thank the referee for remarking to us that the number of abundant numbers given above in the intervals of size $10^{7}$ are compatible with a binomial law with parameters $m_{p}=2476200$ and $s=1365$.

\section{REFERENCES}

[Behrend 1933] F. Behrend, "Über Numeri abundantes, II", Sitzungsber. Preuss. Akad. Wiss. (1933), 280293.

[Davenport 1933] H. Davenport, "Über Numeri abundantes", Sitzungsber. Preuss. Akad. Wiss. (1933), 830-837. 
[Deléglise and Nicolas 1994] M. Deléglise and J.-L. Nicolas, "Sur les entiers inférieurs à $x$ ayant plus de $\log (x)$ diviseurs", J. Théor. Nombres Bordeaux 6:2 (1994), 327-357.

[Elliott 1979] P. D. T. A. Elliott, Probabilistic number theory, I: Mean-value theorems, Grundlehren der Mathematischen Wissenschaften, Springer, New York, 1979.

[Glaisher 1891] W. L. Glaisher, "On the sums of the inverse powers of the prime numbers", Quaterly Journal of Math. 25 (1891), 347-362.

[Martinet et al. 1973] J. Martinet, J. M. Deshouillers, and H. Cohen, "La fonction somme des diviseurs", pp. Exp. No. 11 in Séminaire de Théorie des Nombres, 1972-1973 (Univ. Bordeaux I, Talence), Lab. Théorie des Nombres, Centre Nat. Recherche Sci., Talence, 1973.

[Tenenbaum 1995] G. Tenenbaum, Introduction à la théorie analytique et probabiliste des nombres, 2nd ed., Cours spécialisés 1, Soc. math. France, Paris, 1995.

[Wall 1972] C. R. Wall, "Density bounds for the sum of divisors function", pp. 283-287 in The theory of arithmetic functions (Kalamazoo, Mich., 1971), edited by A. A. Gioia and D. L. Goldsmith, Lecture Notes in Math. 251, Springer, Berlin, 1972.

Marc Deléglise, Institut Girard Desargues (UPRES-A 5028), Département de Mathématiques, Université Lyon 1, 43 Bld du 11 Novembre 1918, 69622 Villeurbanne cedex, France (deleglis@desargues.univ-lyon1.fr)

Received January 21, 1997; accepted August 11, 1997 